\title{
Gravitational Deflection of Celestial Bodies and Photons
}

\author{
Barbaro Quintero-Leyva \\ Independent Work, Miami, FL, USA \\ Email: doserate2002@yahoo.com
}

How to cite this paper: Quintero-Leyva, B. (2018) Gravitational Deflection of Celestial Bodies and Photons. Open Access Library Journal, 5: e4452.

https://doi.org/10.4236/oalib.1104452

Received: February 27, 2018

Accepted: March 24, 2018

Published: March 27, 2018

Copyright (c) 2018 by author and Open Access Library Inc.

This work is licensed under the Creative Commons Attribution International License (CC BY 4.0).

http://creativecommons.org/licenses/by/4.0/

\begin{abstract}
The gravitational deflection angle of celestial bodies travelling near the sun with large eccentricity was derived using the extended Newtonian theory (ENET) and Einstein general theory of relativity (GTR). It was found that the non-Newtonian gravitational deflection of celestial bodies for ENET is 1.5 times the prediction of GTR. The deflection angle of the photon however coincided with the light deflection of GTR. It was also found that the photon's gravitational deflection obeys (as in GTR) an ODE which is a special case of the one for relativistic celestial bodies.
\end{abstract}

\section{Subject Areas}

Classical Physics, Modern Physics, Particle Physics, Special Theory of Relativity

\section{Keywords}

Celestial Mechanics, Newtonian Gravitation, Newton's $2^{\text {nd }}$ Law, Theory of Relativity, Perihelion Precession, Gravitational Deflection, Cosmology

\section{Introduction}

The gravitational deflection of relativistic (speed being a significant fraction of the speed of light) celestial bodies cannot accurately be described with the Newtonian theory as the Newtonian concepts of time interval and space interval need to be modified to accommodate the Michelson-Morley experiment's result.

Einstein in 1915 [1] developed the so called General Theory of Relativity (GTR) in spite of having developed 10 years earlier the special theory of relativity (STR) [2]. The GTR assumes a curved space-time concept which needs the use of tensors. It, however, has repeatedly been experimentally confirmed in many 
instances and if dark matter really exists it has not been falsified either. That theory explains satisfactorily the remarkable observation of Le Verrier regarding a remaining precession of the perihelion of Mercury after the influence of other planets and other effects are considered. GTR also predicted that, the gravitational deflection of light should be twice the one derived by Sodner [3] using Newtonian theory (in a previous paper however Einstein derived a light deflection consistent with the results of Sodner [4]). It is noted that the GTR ODE (in polar coordinates) for celestial bodies and for light are not the same and the concept of null geodesic is used to justify the transition from one ODE to the other one [5].

Many Alternative theories to the GTR that account for the deflection of light by the Sun can be found in the literature, few examples are: Modified relativistic theory of gravitation with focus on the Mach's principle [6]; The generalization of STR to an acceleration field [7]; Simulation model of light that involves only the gravitational red-shift factor [8]; Explanation of the light deflection based on classical electromagnetic theory [9].

The objective of this work is to determine the gravitational deflection angle of celestial bodies while traveling in the neighborhood of the Sun at relativistic speed using ENET. The photon's gravitational deflection is considered (as in GTR) to obey an ODE which is special case of the one used for celestial bodies. It was found, unexpectedly, that the non-Newtonian gravitational deflection of celestial bodies (with significant large eccentricity) near the sun for ENET is 1.5 times the prediction of GTR. The deflection angle of photons however coincided with the light deflection of GTR.

This work is considered important for several reasons, among them:

- ENET is mathematically simple. It keeps the vector calculus as the essential mathematical tool. This provides computational advantage (in comparison with the tensor theory of GTR) in problems regarding, for example, the gravitational $\mathrm{N}$-body-problem of celestial bodies when $\mathrm{N}$ is large.

- It stresses the need of treating (mathematically and therefore physically) the photon differently from the celestial bodies (as in GTR). Note that some authors extend the equation of the light deflection of the photon to celestial bodies without any distinction.

- The fact that the photon was needed to be treated differently from the celestial bodies could imply that the relativistic equation of motion as described here could have a deeper meaning in nature.

- It was found that the non-Newtonian gravitational deflection of relativistic celestial bodies according to ENET is significantly different from the GTR's result. Measurements of the deflection angle could provide a falsification of ENET and/or GTR. The author is not aware of experimental results regarding the gravitational deflection angle of relativistic celestial bodies.

The rest of this paper is structured as follows: Section 2 briefly describes the fundament of ENET and calculates the deflection angle of significantly massive 
bodies (celestial bodies) using ENET and GTR; Section 3 describes the need of GTR to treat the photon differently from the celestial bodies and shows how, using ENET, the deflection angle of the photon is empirically obtained; In Section 4 some remarks are made concerning the concepts of force, mass, and acceleration in the context of STR, electron theory and ENET. Section 5 provides the summary and concluding remarks.

\section{Gravitational Deflection of Relativistic Celestial Bodies}

The Michelson-Morley experiment result along with the Lorentz-Fitzgerald transformation, and Einstein special theory of relativity support the concept of the apparent time dilation and length contraction given by

$\Delta t=\Delta t^{\prime}\left(1-\beta^{2}\right)^{-1 / 2}:$ Time dilation; $\Delta x=\Delta x^{\prime}\left(1-\beta^{2}\right)^{1 / 2}$ : Length contraction; $\beta=v / c$

$v$. Is the speed of the moving reference frame and $c$ is the speed of light in vacuum.

The concept of acceleration requires 2 space-time intervals (contraction/dilation: two successive-interval boosts) to relate 3 space-time events. Considering another reference frame moving with a velocity $v$ with respect to the prime reference frame, the following can be written for the acceleration in one Cartesian direction [10]:

$$
a=\left.\left(1-\beta^{2}\right)^{3} \frac{\mathrm{d} v}{\mathrm{~d} t}\right|_{N}
$$

$\left.\frac{\mathrm{d} v}{\mathrm{~d} t}\right|_{N}:$ Newtonian acceleration. $v$. Is the speed of the moving object used in $\beta$ also.

So the extended Newton's $2^{\text {nd }}$ law can be written as

$$
F=m a \Rightarrow F=\left.m\left(1-\beta^{2}\right)^{3} \frac{\mathrm{d} v}{\mathrm{~d} t}\right|_{N}
$$

In gravitational bound systems the moving object is revolving around a very massive body or around the center of mass of the system, the direction of the moving body in question is continuously changing, assuming that the length contraction happens in the 3 Cartesian directions, Equation (1) can be applicable to multidimensional motion [10]. It is noted that no physical arguments against the application of Equation (1) to gravitational unbound motions of uncharged massive objects are conceived.

The balance between the force given by the extended Newton's $2^{\text {nd }}$ law (Equation (2)) and the Newtonian gravitation in polar coordinates was obtained in [10] for the solar system (Heliocentric coordinate), that equation generalized for an arbitrary exponent was written as

$$
\frac{\mathrm{d}^{2} u}{\mathrm{~d} \theta^{2}}+u=b\left(1-\lambda\left[\left(\frac{\mathrm{d} u}{\mathrm{~d} \theta}\right)^{2}+u^{2}\right]\right)^{-n}
$$




$$
b=G(M+m) / h^{2}, \lambda=h^{2} / c^{2}
$$

Note that Equation (3) can be obtained from $F=\left.m\left(1-\beta^{2}\right)^{n} \frac{\mathrm{d} v}{\mathrm{~d} t}\right|_{N}$.

The solution of Equation (3), noting that the multiplier of $b$ represents a small perturbation, can be expressed in terms of a Fourier series as [5]

$$
u=b+\lambda \beta_{0}+b e \cos (\rho \theta)+\lambda \sum_{2}^{\infty} \beta_{v} \cos (v \rho \theta)
$$

From Equation (4) the following is obtained 10]:

$$
\begin{gathered}
\frac{\mathrm{d} u}{\mathrm{~d} \theta}=-b e \rho \sin (\rho \theta)-\lambda \rho \sum_{2}^{\infty} \beta_{v} v \sin (v \rho \theta) \\
\frac{\mathrm{d}^{2} u}{\mathrm{~d} \theta^{2}}=-b e \rho^{2} \cos (\rho \theta)-\lambda \rho^{2} \sum_{2}^{\infty} \beta_{v} v^{2} \cos (v \rho \theta) \\
\lambda u^{2}=\lambda b^{2}\left(1+e^{2} / 2+2 e \cos (\rho \theta)+e^{2} / 2 \cos (2 \rho \theta)\right) \\
\lambda\left(\frac{\mathrm{d} u}{\mathrm{~d} \theta}\right)^{2}=\frac{1}{2} \lambda(b e \rho)^{2}-\frac{1}{2} \lambda(b e \rho)^{2} \cos (2 \rho \theta) \\
\lambda\left[u^{2}+\left(\frac{\mathrm{d} u}{\mathrm{~d} \theta}\right)^{2}\right]=\lambda\left[a_{1}+a_{2} \cos (\rho \theta)+a_{3} \cos (2 \rho \theta)\right] \\
a_{1}=(b e \rho)^{2} / 2+b^{2}\left(1+e^{2} / 2\right), a_{2}=2 b^{2} e, a_{3}=-(b e \rho)^{2} / 2+b^{2} e^{2} / 2
\end{gathered}
$$

For $n=+3$,

$$
\left(1-\lambda\left[\left(\frac{\mathrm{d} u}{\mathrm{~d} \theta}\right)^{2}+u^{2}\right]\right)^{-3}=1 /\left(1-3 \lambda a_{1}-3 \lambda a_{2} \cos (\rho \theta)-3 \lambda a_{3} \cos (2 \rho \theta)\right)
$$

Substituting the above equations into Equation (3), neglecting the terms containing $2^{\text {nd }}$ and higher power of $\lambda$ and equating the coefficients of $\cos (\rho \theta), \quad \rho \approx 1-3 \lambda b^{2}$ is obtained which yields the Einstein's GTR results for the intrinsic (two body problem) perihelion precession of the planets [10]:

Similarly, writing Equation (4) until the term $\cos (3 \rho \theta)$ and substituting into Equation (3), the following is obtained:

$$
\begin{aligned}
& \beta_{0}=3 a_{1} b+\frac{3}{2} a_{2}\left(-b e \rho^{2}+b e\right), \\
& \beta_{2}=\frac{\frac{3}{2} a_{2}\left(-b e \rho^{2}+b e\right)+3 a_{3} b}{1-4 \rho^{2}}, \\
& \beta_{3}=\frac{\frac{3}{2} a_{3}\left(-b e \rho^{2}+b e\right)}{1-9 \rho^{2}}
\end{aligned}
$$

Making $u=0$ (large $r$ ) in Equation (4) and expanding in Taylor series around $\frac{\pi}{2}$ (reference angle) up to the linear term: 


$$
\begin{aligned}
& b+\lambda \beta_{0}+b e\left(\cos \left(\rho \frac{\pi}{2}\right)+\rho \sin \left(\rho \frac{\pi}{2}\right) \frac{\pi}{2}\right)-b e \rho \sin \left(\rho \frac{\pi}{2}\right) \theta \\
& +\lambda \beta_{2}(\cos (\rho \pi)+\rho \sin (\rho \pi) \pi)-2^{2} \lambda \beta_{2} \rho \sin (\rho \pi) \theta \\
& +\lambda \beta_{3}\left(\cos \left(\frac{3}{2} \rho \pi\right)+\frac{3}{2} \rho \pi \sin \left(\frac{3}{2} \rho \pi\right)\right)-3^{2} \lambda \beta_{3} \rho \sin \left(\frac{3}{2} \rho \pi\right) \theta=0
\end{aligned}
$$

From Equation (5) (or from an upgraded equation that could include higher terms and or a different reference angle) $\theta$ can be calculated for any value of the eccentricity. For the special case of $e \gg 1$ :

$$
\rho=1, \beta_{0}=3 b^{3} e^{2}, \beta_{2}=0, \beta_{3}=0 \Rightarrow \theta=1 / e+3 \lambda b^{2} e+\frac{\pi}{2}
$$

Defining the deflection angle as

$$
\delta \equiv-\pi+2 \theta \Rightarrow \delta=2 / e+6 \lambda b^{2} e
$$

The GTR equation of motion in polar coordinates can be written as [5]:

$$
\frac{\mathrm{d}^{2} u}{\mathrm{~d} \theta^{2}}+u=\frac{G M}{h^{2}}+3 \frac{G M}{c^{2}} u^{2}
$$

The speed of light was explicitly written here for convenience.

Equation (7) for a heliocentric coordinate system using the notation of Equation (3) can be written as

$$
\begin{gathered}
\frac{\mathrm{d}^{2} u}{\mathrm{~d} \theta^{2}}+u=b_{E}\left(1+\lambda_{E} u^{2}\right) \\
b_{E} \approx \frac{G(M+m)}{h^{2}}=b, \lambda_{E}=3 \frac{h^{2}}{c^{2}}=3 \lambda
\end{gathered}
$$

Following the same methodology used to get Equation (6) the following was obtained

$$
\delta_{E}=2 / e+\frac{4}{3} \lambda_{E} b^{2} e=2 / e+4 \lambda b^{2} e
$$

Note from Equations (6) and (9) that the non-Newtonian deflection of celestial bodies for ENET is 1.5 times that of the GTR.

It is curious that even though Equations (3) and (7) yield the same perihelion precession of the planets (for the level of accuracy demanded), Equations (6) and (9) yield different deflection angles. It could therefore be worthy to design experiments to determine $\mathrm{n}$ in the equation $\delta=2 / e+2 n \lambda b^{2} e$.

\section{Photon Deflection}

It is mathematically reasonable to expect that even for particles with very small mass, Equations (3) and 7(8) would be still valid. However for the special case of the photons (considered by many researchers as being gravitational massless even though they have electromagnetic mass) the measured deflection angle of the photons coming from stars optically near the Sun is not in agreement with the results of the Equations (6) and (9) as shown below: 


$$
\text { For } e \gg 1 \Rightarrow e \approx \frac{R_{\text {sun }} c^{2}}{G M_{\text {sun }}}
$$

Equation (6) (ENET) yields $\delta=2 / e+6 \lambda b^{2} e=\frac{8 G M_{\text {sun }}}{R_{\text {sun }} c^{2}}=4 \delta_{\text {Sodner }} \Rightarrow$ twice the measured value

$$
\left(\delta_{\text {measured }}=2 \delta_{\text {Sodner }} \approx 1.75 "\right)[11]
$$

Equation (9) (GTR) yields $\delta_{E}=2 / e+4 \lambda b^{2} e=\frac{6 G M_{\text {sun }}}{R_{\text {sun }} c^{2}}=3 \delta_{\text {sodner }} \Rightarrow 1.5$ the measured value

GTR describes the equation of motion of the light with a different ODE requiring that the light trajectory be a null geodesic of the curved space-time concept [5]. The resultant ODE is

$$
\left(\frac{\mathrm{d} u}{\mathrm{~d} \theta}\right)^{2}+u^{2}=\frac{k^{2}}{h^{2}}+2 G M \cdot u^{3}
$$

where, $k$ is a constant.

Based on this ODE the correct deflection angle equation was obtained in [5].

By taking the derivative of Equation (10) with respect to $\theta$ the following ODE is obtained:

$$
\frac{\mathrm{d}^{2} u}{\mathrm{~d} \theta^{2}}+u=3 \frac{G M}{c^{2}} u^{2}
$$

Equation (11) also yields the correct deflection angle [12]. Note that eq. 11 is a special case of Equation 7(8). It is curious that the difference between both equations is just $G M / h^{2}$ which contains the angular momentum per unit of mass of the photon. If $h$ is assumed to be infinite for a massless photon, that could provide a natural transition to Equation (11). It is noted that reference [9] provided the same argument to this issue. Notice however that applying the Einstein equation (STR) to the photon, a mass given by $m_{p h}=E_{p h} / c_{p h}^{2}$ could be calculated. It is noted however that $c_{p h}$ is considered here to be very close to $c$ but it is not assumed to be a constant.

Based on the arguments just given, it was thought that a special case of Equation (3) could perhaps yield the correct value of the gravitational deflection of the photon. It turned out that for $n=1$ that is the case as is shown next.

The correcting multiplier on the RHS of Equation (3) for positive n, after neglecting $2^{\text {nd }}$ and higher powers $\lambda$ can be written as

$$
\left(1-\lambda\left[\left(\frac{\mathrm{d} u}{\mathrm{~d} \theta}\right)^{2}+u^{2}\right]\right)^{-n}=1 / d_{n}
$$

with $d_{n}=1-n \lambda a_{1}-n \lambda a_{2} \cos (\rho \theta)-n \lambda a_{3} \cos (2 \rho \theta)$

Substituting into Equation (3), the following is obtained:

$$
\beta_{0}=n a_{1} b+\frac{n}{2} a_{2}\left(-b e \rho^{2}+b e\right)
$$




$$
\begin{gathered}
\beta_{2}=\frac{\frac{n}{2} a_{2}\left(-b e \rho^{2}+b e\right)+n a_{3} b}{1-4 \rho^{2}} \\
\beta_{3}=\frac{\frac{n}{2} a_{3}\left(-b e \rho^{2}+b e\right)}{1-9 \rho^{2}} \\
\text { For } \rho=1: \quad \beta_{0}=n a_{1} b, \beta_{2}=-\frac{n a_{3} b}{3}=0, \beta_{3}=0
\end{gathered}
$$

Substituting into Equation (5):

$$
\theta=\frac{\pi}{2}+\frac{1}{e}+\lambda \frac{n a_{1}}{e}, \text { for } e \gg 1 \Rightarrow \delta=\frac{2}{e}+2 n \lambda b^{2} e
$$

For $n=1 \Rightarrow \delta=\frac{2}{e}+2 \lambda b^{2} e=\frac{4 G M_{\text {sun }}}{R_{\text {sun }} c^{2}}$ in agreement with experiments.

It is noted that $n=1$ can be obtained by applying one Lorentz time-dilation boost (correction) to the concept of the classical time interval in the Newtonian acceleration (no length contraction for photons). So for the case of the photon:

$$
a=\left.\left(1-\beta^{2}\right) \frac{\mathrm{d} v}{\mathrm{~d} t}\right|_{N}
$$

When equating Equation (12) to the Newtonian Gravitational law, the equation of motion of the photon cannot be solved numerically unless the initial velocity of the photon (initial condition) does not coincide with the speed of light or the limiting speed in the Lorentz factor is not exactly $c$. This suggests that the Lorentz factor for the photon should not be exactly the same as the one for celestial bodies despite that for time independent calculation (Equation (3) with $n$ $=1$ ) the correct deflection angle is obtained.

It is hoped that $n=1$ could also yield a gravitational time delay consistent with the Shapiro time delay. It is also hoped that an extended Newtonian's gravitational law (perhaps similar to electromagnetism as previously attempted by, for example, Maxwell and Heaviside) combined with ENET (extended Newton's $2^{\text {nd }}$ law), could yield gravitational waves consistent with the recent LIGO discovery and with the orbital period decay of binary systems previously reported.

\section{Some Remarks on Force, Mass and Acceleration}

If the Planck equation $\frac{\mathrm{d}}{\mathrm{d} t}\left(\frac{m \vec{v}}{\sqrt{1-\beta^{2}}}\right)$ is used as the concept of inertial force, the following is obtained as special cases [5]

$$
F=\left.m\left(1-\beta^{2}\right)^{-3 / 2} \frac{\mathrm{d} v}{\mathrm{~d} t}\right|_{N} \quad F=\left.m\left(1-\beta^{2}\right)^{-1 / 2} \frac{\mathrm{d} v}{\mathrm{~d} t}\right|_{N}
$$

$n=-3 / 2$ applies when the acceleration is parallel to the velocity and $n=-1 / 2$ when the force is perpendicular to the velocity (these relations were also obtained by Lorentz and Einstein). Those values of $n$ however, do not yield the correct angular deflection and neither the correct sign and magnitude of the pe- 
rihelion precession of the planets [13]. Note that for those values of $n$ the apparent variation of mass with the speed (a concept usually attributed to electron theory and STR) is drastically different from the celestial bodies $(n=3)$, while in the former cases the mass apparently increases with the speed, in the latter one the mass apparently decreases with the speed. Notice however, that in ENET it is not the mass that changes with the speed, but it is the relativistic acceleration that depends on the speed.

It is noted that the equation for $n=+3$, not derivable from the Planck equation, has been obtained in [14] (assuming that the speed of the body is much smaller than the speed of light in vacuum), as well as in [15] where the gravitational and the inertial mass was considered to correspond to $n=-3 / 2$ (longitudinal mass) in problems of elementary particles confinement by the gravitational force.

The special cases just mentioned $(n=-3 / 2,-1 / 2)$ could be checked in experiments using the principles of particle accelerators after correcting for radiation effects.

If the concept of inertial acceleration is independent of the type of force, it is expected that if those special cases are verified on particle accelerators, they should also be valid for gravitational force (for example, in head on motions and in approximately circular orbits). If that would not be the case then the acceleration should also depend on other properties/conditions as, for example, on the electric charge (net or distribution), on a mass lower limit (case of the photon?), etc. If the relativistic acceleration does depends on some properties of the particle in question the exponent in Equation (3) (expressible as a power of the Lorentz factor) has a deep meaning in nature.

It is believed that more attention needs to be paid to the concept of force and acceleration in experimental physics.

It could be worthy to design experiments to determine $\mathrm{n}$ in motions under gravitational and/or electric/magnetic forces (i.e. outer space gravitational/electric deflection of charged/uncharged particles/bodies, charged/uncharged particle scattering/diffraction with and without gravitational effects, etc.) to determine, for example, $n$ and its potential dependence on the properties and motion conditions of the object/particle in question.

\section{Summary and Concluding Remarks}

The gravitational deflection angle of relativistic celestial bodies travelling near the Sun with large eccentricity was derived using ENET and GTR.

It was found that the non-Newtonian gravitational deflection of celestial bodies (with large eccentricity) using ENET is 1.5 times the prediction of GTR.

The deflection angle of the photon for ENET however coincided with the light deflection angle of GTR.

The photon gravitational deflection for ENET obeys an ODE which is a special case of the one used for relativistic celestial bodies. This is the case also in GTR.

It could be worthy to determine, experimentally, the value of $n$ in Equation (3). 


\section{Acknowledgements}

I would like to thank Professor C. G. Vayenas for helpful discussions.

\section{References}

[1] Einstein, A. (1916) The Foundation of the Generalized Theory of Relativity (Die Grundlage der allgemeinen Relativitatstheorie). Annalen der Physik, 354, 769-822. https://doi.org/10.1002/andp.19163540702

[2] Einstein, A. (1905) On the Electrodynamics of Moving Bodies. English translation from "Zur elektrodynamik bewegter Korper", Prepared by John Walker (1999). Annalen der Physic, 17, 891-921. https://doi.org/10.1002/andp.19053221004

[3] Soldner J. (1801) On the Deflection of Light Ray from Its Rectilinear Motion, by the Attraction of a Celestial Body at Which It Nearly Passes by. English translation downloaded from https://en.wikisource.org/w/index.php

[4] Einstein A. (1911) On the Influence of Gravitation on the Propagation Light. Annalen der Physik, 35, 898-908. (English translation by Michael D. Godfrey) https://doi.org/10.1002/andp.19113401005

[5] Bergmann P.G. (1976) Introduction to the Theory of Relativity. Dover Publications, Inc., New York.

[6] Brans, C. and Dicke, R.H. (1961) Mach's principle and a relativistic theory of gravitation. Physical Review, 124, 925. https://doi.org/10.1103/PhysRev.124.925

[7] Note, M. (1985) The Special Theory of Relativity in a Gravitational Field. International Journal of Fusion Energy, 3, No. 2.

[8] Biswas, A. and Mani, K. (2004) Simulation Model for Shapiro Time Delay and Light Deflection Experiments. CEJP, 2, 687-697. https://doi.org/10.2478/BF02475569

[9] Hajra S. (2012) Classical Interpretation of Relativistic Phenomena. Journal of Modern Physics, 3, 187-199. https://doi.org/10.4236/jmp.2012.32026

[10] Quintero-Leyva, B. (2016) An Extended Newtonian Theory for Gravitational Bound Systems. Open Access Library Journal, 3, e2678. https://doi.org/10.4236/oalib.1102678

[11] Will, C.M. (2015) The 1919 Measurement of the Deflection of Light. Classical and Quantum Gravity, 32, Article ID: 124001

[12] Prakash S. (2000) Relativistic Mechanics. Pragati Prakashan, Begum Bridge. Meerut-25001, UP, India, 442-444.

[13] Quintero-Leyva, B. (2015) On the Intrinsic Precession of the Perihelion of Mercury. Open Access Library Journal, 2, e2239. https://doi.org/10.4236/oalib.1102239

[14] Hajra S. (2014) Classical Interpretation of Relativistic Precessions. Chinese Physics B, 23, 040402. https://doi.org/10.1088/1674-1056/23/4/040402

[15] Vayenas, C.G. and Sountie, S.N.-A. (2012) Gravity, Special Relativity and the Strong Force. A Bohr-Einstein-de Broglie Model for the Formation of Hadrons. Springer. https://doi.org/10.1007/978-1-4614-3936-3 